Journal of the Operations Research

Society of Japan

Vol. 42, No. 2, June 1999

\title{
A LOGICAL INTERPRETATION FOR THE EIGENVALUE METHOD IN AHP
}

\author{
Kazuyuki Sekitani Naokazu Yamaki \\ Shizuoka University
}

(Received October 1, 1998)

\begin{abstract}
The eigenvalue method (EM), that is to find the principal eigenvector of a pairwise comparison matrix, is widely used and known to be practical in Analytic Hierarchy Process (AHP). However, the validity of EM has never been fully proved. In this article, we present an equilibrium model and four optimization models to show the logical justification for using EM in AHP. By introducing two concepts, self-evaluation and non-self-evaluation, into AHP, the fundamental theorem (Frobenius' Theorem) for EM is interpreted as two optimization problems. From these two concepts, a noncooperative game with a pairwise comparison matrix is also formulated and its equilibrium solution is the principal eigenvector. We propose two discrepancy indices between self-evaluation and non-self-evaluation and formulate four discrepancyminimization problems. An optimal solution for two minimization problems among them is equal to the principal eigenvector.
\end{abstract}

\section{Introduction}

Since Saaty [7] developed the Analytic Hierarchy Process (AHP) in the 1970s, AHP has been widely used and applied to decision problems in various situations such as business and social economic planning (see for example [13], [15] and [18]).

Saaty [7] has developed the eigenvalue method (EM) in order to synthesize a pairwise comparison matrix $A$ and to obtain a priority weight vector for several decision criteria and alternatives. Here an eigenvector of the matrix $A$ is used for the priority weight vector. Two other methods: the logarithmic least square method (LLSM) [4] and the least square method (LSM) [3], are also proposed for their priority weight vectors. These methods are compared with EM in several literatures (see for example [6], [8] and [9]).

In EM the priority weight vector is set to the right principal eigenvector $w$ of the pairwise comparison matrix $A$. Hence EM is to find the maximum value $\lambda$ and its corresponding vector $w$ such that $A w=\lambda w$. Saaty [8] and Harker and Vargas [6] assert that the priority weight vector of $\mathrm{EM}$ is given by

$$
w=\lim _{k \rightarrow \infty} \frac{A^{k} e}{e^{T} A^{k} e},
$$

where $e$ is a vector of ones and the superscript "T" indicates a vector transpose. They interpret the priority weight vector as the convergent state (1.1) of the averaging process $\left\{\left(A^{k} e / e^{T} A^{k} e\right) \mid k=1,2, \ldots\right\}$ by means of a directed graph. For a pairwise comparison matrix $A=\left(a_{i j}\right)$ of order $n$, the directed graph is formed by a node set $I=\{1, \ldots, n\}$ and an arc set $\{(i, j) \mid i \in I, j \in I$ and $i \neq j\}$ such that the arc $(i, j)$ from the node $i$ to the node $j$ is labeled as $a_{i j}$. From the graph-theoretical argument they conclude that EM will retain rank ordering in an inconsistent matrix (see for details [6], [8] and [10]).

As suggested in [11], if for an inconsistent pairwise comparison matrix $A$ there is an unknown consistent matrix $\bar{A}$ such that $A$ is slightly perturbed from $\bar{A}$, the priority weight 
vector of $\bar{A}$ will be considered an ideal weight vector of $A$ and EM will give an approximation weight vector for the ideal weight vector.

On the other hand, as described in [1], [11] and [14], LLSM and LSM are based on the following optimization problems. With the input data of the pairwise comparison matrix $A$ of order $n$, a priority weight vector $w=\left(w_{1}, \ldots, w_{n}\right)^{T}$ is set to a solution for one of the two optimization problems:

$$
\begin{array}{lll}
\min . & \sum_{i=1}^{n} \sum_{j=1}^{n}\left|\log a_{i j}-\log \left(w_{i} / w_{j}\right)\right|^{2} & \text { for LLSM and } \\
\min . & \sum_{i=1}^{n} \sum_{j=1}^{n}\left|a_{i j}-w_{i} / w_{j}\right|^{2} & \text { for LSM. }
\end{array}
$$

It is because they assume the error models $a_{i j}=\left(w_{i} / w_{j}\right) \epsilon_{i j}$ for LLSM or $a_{i j}=\left(w_{i} / w_{j}\right)+$ $\epsilon_{i j}$ for LSM, where the error $\epsilon_{i j}$ has some continuous distribution.

The aim of this study is to give a logical justification for EM by means of optimization/equilibrium models. The study of AHP based on these models has the following two advantages:

- one can clarify the conditions of EM in terms of the model-based AHP, which are implicitly assumed in the convergence for the averaging process of (1.1) and

- one can separate the three processes: modeling process for the practical problem, estimating process of a priority weight vector and analyzing process for the relationship between the priority weight vector and the pairwise comparison matrix or the structure of the model. Hence, for example, a priori constraints of priority weight vector will be incorporated in the model of AHP (see for example [16]). After solving the model and obtaining a priority weight vector, we could analyze the sensitivity of the priority weight vector for the pairwise comparison matrix $A$ and the additional constraints.

The structure of this article is organized in the following manner. In section 2 two new concepts "self-evaluation" and "non-self-evaluation" are introduced. Then the two wellknown theorems, "Frobenius' Theorem" and "Perron-Frobenius' Theorem", are interpreted as the statements of a relationship between two optimization problems which are based on the proposed concepts. These two theorems guarantee the convergence of EM and the existence of its solution. Then we show in section 3 that our framework of AHP can be naturally interpreted as a noncooperative game, and that the priority weight vector of EM satisfies an equilibrium condition of the game. Section 4 introduces the discrepancy index between self-evaluation and non-self-evaluation, formulates some optimization problems for minimizing the discrepancy indices, and analyzes the relationship between the eigenvector and the priority weight vector for each optimization problem. Conclusions and further extensions of our model-based AHP are summarized in Section 5.

\section{Fundamental Theorems and Basic Concepts}

Consider the problem of comparing a set of $n$ alternatives with respect to a single criterion. Then, the size of the pairwise comparison matrix $A$ is $n$. Generally the number $n$ of alternatives is not less than 3 and all pairwise comparisons between distinct alternatives are done with respect to either dominance, importance or preference. Here, we simply call them "value." Each entry $a_{i j}$ of the pairwise comparison matrix $A$ represents the ratio of the value of the $i^{\text {th }}$ alternative to one unit of the value of the $j^{\text {th }}$ alternative. This study assumes that $a_{i j}>0$ and $a_{i i}=1$ for all $i, j \in I$. As described in [8], the observed value 
in practice does not necessarily satisfy that $a_{i j}=1 / a_{j i}$. Therefore, we do not assume that $a_{i j}=1 / a_{j i}$ for all $i, j \in I$. Hereafter the pairwise comparison matrix $A$ is a positive matrix with the unit diagonal entry $a_{i i}=1$ for all $i \in I$, but not necessarily reciprocal.

Our model-based AHP supposes that every alternative evaluates itself, and that it gives itself a positive real number. Let $w_{i}$ be the positive real number given to the $i^{\text {th }}$ alternative by itself. The value $w_{i}$ of the $i^{t h}$ alternative is called the $i^{t h}$ self-evaluation value. The value $a_{i j} w_{j}$ represents the evaluation value of the $i^{\text {th }}$ alternative from the viewpoint of the $j^{\text {th }}$ alternative 'when the $j^{\text {th }}$ self-evaluation value is $w_{j}$. Averaging $a_{i j} w_{j}$ over $j \neq i$, we obtain $\sum_{j \neq i} a_{i j} w_{j} /(n-1)$, which we call the $i^{\text {th }}$ non-self-evaluation value.

Example 1 Let $n=4$ and consider the self-evaluation vector $w=\left(w_{1}, w_{2}, w_{3}, w_{4}\right)^{T}$ and the pairwise comparison matrix

$$
A=\left(\begin{array}{cccc}
1 & 2 & 4 & 3 \\
1 / 3 & 1 & 3 & 2 \\
1 / 4 & 1 / 3 & 1 & 1 \\
1 / 4 & 1 / 2 & 1 & 1
\end{array}\right)
$$

Then

$$
\begin{aligned}
\text { The first non-self-evaluation value } & =\frac{2 w_{2}+4 w_{3}+3 w_{4}}{3} \\
\text { The second non-self-evaluation value } & =\frac{(1 / 3) w_{1}+3 w_{3}+2 w_{4}}{3} \\
\text { The third non-self-evaluation value } & =\frac{(1 / 4) w_{1}+(1 / 3) w_{2}+w_{4}}{3} \\
\text { The fourth non-self-evaluation value } & =\frac{(1 / 4) w_{1}+(1 / 2) w_{2}+w_{3}}{3} .
\end{aligned}
$$

We will introduce some mathematical terminologies and notations used in the later theorems and lemmas. We denote the zero vector by 0 . A vector $w$ whose elements are all positive is called positive and we denote it by $w>0$. A nonnegative matrix $A$ is called irreducible if the direct graph with a node set $I$ and an arc set $\left\{(i, j) \mid a_{i j}>0\right.$ and $\left.i \neq j\right\}$ is strongly connected.

The following well-known theorem guarantees that a priority vector of EM is unique, and that it is positive.

Theorem 1 (Perron-Frobenius' Theorem [12]) Suppose that $A$ is an irreducible nonnegative matrix. Then there are an eigenvalue $\lambda$ and the corresponding eigenvector $w$ satisfying the following two conditions:

(1) $A w=\lambda w, \lambda>0, w>0$ and $\lambda \geq|\alpha|$ for every eigenvalue $\alpha$ of the matrix $A$.

(2) $\lambda$ is a single root of the characteristic equation of $A$.

We denote an eigenspace of the matrix $A$ corresponding to the eigenvalue $\lambda$ by $M(\lambda: A)$, that is $M(\lambda: A)=\{w \mid A w=\lambda w\}$. Note that $\operatorname{dim} M(\lambda: A)=1$ if the eigenvalue $\lambda$ is a single root of the characteristic equation of $A$. (When an eigenvalue is a single root of the characteristic equation of the matrix, it is geometrically and algebraically simple. see for detail [2].) An eigenvector $w \in M(\lambda: A)$ corresponding to the simple $\lambda$ is called unique. From theses terminologies and notations we represent Theorem 1 as the following corollary. Corollary 2 Suppose that $A$ is an irreducible nonnegative matrix. Let $\lambda_{\text {max }}$ be the principal eigenvalue of $A$. Then, $\lambda_{\max }>0$ and $\operatorname{dim} M\left(\lambda_{\max }: A\right)=1$. Furthermore for all nonzero vector $w \in M\left(\lambda_{\max }: A\right), w>0$ or $w<0$. 
Let $a_{i}$ be the $i^{\text {th }}$ row vector of $A$, then any eigenvector $w$ of $A$ corresponding to the eigenvalue $\lambda$ satisfies $a_{i} w=\lambda w_{i}$ for all $i \in I$. Corollary 2 implies that

$$
\frac{a_{i} w}{w_{i}}=\lambda_{\max } \quad \text { for all } i \in I
$$

where $\lambda_{\max }$ is the principal eigenvalue of the irreducible nonnegative matrix $A$ and $w \in$ $M\left(\lambda_{\max }: A\right)$. The following theorem based on (2.1) describes some important properties of the principal eigenvalue and the corresponding eigenvector of the matrix $A$.

Theorem 3 (Frobenius' Theorem [5]) Suppose that $A$ is a nonnegative matrix, and that $\lambda_{\max }$ is the principal eigenvalue of $A$. Then for every $n$-dimensional positive vector $w$,

$$
\min \left\{\frac{a_{1} w}{w_{1}}, \ldots, \frac{a_{n} w}{w_{n}}\right\} \leq \lambda_{\max } \leq \max \left\{\frac{a_{1} w}{w_{1}}, \ldots, \frac{a_{n} w}{w_{n}}\right\}
$$

Furthermore, if the matrix $A$ is irreducible,

$$
\max _{w>0} \min \left\{\frac{a_{1} w}{w_{1}}, \ldots, \frac{a_{n} w}{w_{n}}\right\}=\lambda_{\max }=\min _{w>0} \max \left\{\frac{a_{1} w}{w_{1}}, \ldots, \frac{a_{n} w}{w_{n}}\right\}
$$

where the two equalities in (2.3) hold for every positive eigenvector $w \in M\left(\lambda_{\max }: A\right)$.

Corollary 4 Suppose that $A$ is an irreducible nonnegative matrix, and that $\lambda_{\max }$ is the principal eigenvalue of $A$. A positive vector attaining the two equalities of (2.3) is a positive eigenvector of $A$ corresponding to $\lambda_{\max }$, and vice versa.

Proof: Suppose that a positive vector $\bar{w}$ attains the two equalities of (2.3). Then, $\lambda_{\max }=$ $\left(a_{1} \bar{w} / \bar{w}_{1}\right)=\cdots=\left(a_{n} \bar{w} / \bar{w}_{n}\right)$. This means that $A \bar{w}=\lambda_{\max } \bar{w}$. Hence, the positive vector $\bar{w}$ is an eigenvector of $A$ corresponding to $\lambda_{\text {max }}$.

It follows immediately from Theorem 3 that the positive eigenvector of $A$ corresponding to $\lambda_{\max }$ attains the two equalities of $(2.3)$.

The following lemma implies that EM is to find a positive vector $w$ such that $A w=\lambda w$. Lemma 5 Suppose that $A$ is an irreducible nonnegative matrix. Let $\lambda_{\max }$ be the principal eigenvalue of $A$. Then, any positive eigenvector of $A$ belongs to $M\left(\lambda_{\max }: A\right)$.

Proof: Let $\bar{w}$ and $\bar{\lambda}$ be a positive eigenvector of $A$ and the corresponding eigenvalue, respectively. Then

$$
A \bar{w}=\bar{\lambda} \bar{w} .
$$

Suppose that $\bar{w} \notin M\left(\lambda_{\max }: A\right)$. From Corollary 2, the eigenvalue $\bar{\lambda}$ is less than the principal eigenvalue $\lambda_{\max }$ of the matrix $A$. Therefore, it follows from (2.4) that

$$
\begin{aligned}
\lambda_{\max } & >\bar{\lambda} \\
& =\frac{a_{1} \bar{w}}{\bar{w}_{1}}=\cdots=\frac{a_{n} \bar{w}}{\bar{w}_{n}} \\
& =\max \left\{\frac{a_{1} \bar{w}}{\bar{w}_{1}}, \cdots, \frac{a_{n} \bar{w}}{\bar{w}_{n}}\right\},
\end{aligned}
$$

which contradicts $(2.2)$ of Theorem 3 .

For a matrix $A$ we define a matrix of order $n$

$$
\hat{A}=\frac{1}{n-1}(A-E)
$$


where $E$ is the identity matrix of order $n$. In the similar manner to the matrix $A$, we denote the $(i, j)$ entry and the $i^{t h}$ row vector of $\hat{A}$ by $\hat{a}_{i j}$ and $\hat{a}_{i}$, respectively. Then we can represent the $i^{\text {th }}$ non-self-evaluation value as $\hat{a}_{i} w$. Note that all diagonal entries $\hat{a}_{i i}$ of the matrix $\hat{A}$ are zero, and that $\hat{a}_{i j} \neq\left(1 / \hat{a}_{j i}\right)$ for all $i \neq j$ if $A$ is reciprocal.

Lemma 6 Suppose that $A$ is a positive matrix whose diagonal entries are all unit. Then, $\hat{A}$ defined by (2.5) is an irreducible nonnegative matrix.

Proof: $\quad \hat{a}_{i j}=a_{i j} /(n-1)>0$ for every $i \neq j$ and $\hat{a}_{i i}=0$ for all $i \in I$.

Lemma 6 means that for the principal eigenvalue $\hat{\lambda}_{\max }$ of the matrix $\hat{A}$ there is a unique positive eigenvector $\hat{w}$. Therefore, we see from Theorem 3 that for the matrix $\hat{A}$

$$
\max _{w>0} \min \left\{\frac{\hat{a}_{1} w}{w_{1}}, \ldots, \frac{\hat{a}_{n} w}{w_{n}}\right\}=\hat{\lambda}_{\max }=\min _{w>0} \max \left\{\frac{\hat{a}_{1} w}{w_{1}}, \ldots, \frac{\hat{a}_{n} w}{w_{n}}\right\} .
$$

Note that the following two optimization problems underlie (2.6).

$$
\begin{aligned}
& \max _{w>0} \min \left\{\frac{\hat{a}_{1} w}{w_{1}}, \ldots, \frac{\hat{a}_{n} w}{w_{n}}\right\} \\
& \min _{w>0} \max \left\{\frac{\hat{a}_{1} w}{w_{1}}, \ldots, \frac{\hat{a}_{n} w}{w_{n}}\right\} .
\end{aligned}
$$

Every term $\left(\hat{a}_{i} w / w_{i}\right)$ in $(2.7)$ and $(2.8)$ is the ratio between the $i^{\text {th }}$ self-evaluation value $w_{i}$ and non-self-evaluation value $\hat{a}_{i} w$. The optimization problem $(2.7)$ is to maximize the least ratio between self-evaluation value and non-self-evaluation value. The optimization problem (2.8) is to minimize the largest ratio. From (2.6) and Theorem 3 we see that both optimization problems (2.7) and (2.8) have a common optimal solution that is any eigenvector $\hat{w}$ of the matrix $\hat{A}$ corresponding to the principal eigenvalue $\hat{\lambda}_{\text {max }}$. It follows from Corollary 4 that an optimal solution of the problem (2.7) or (2.8) is a positive eigenvector of $M\left(\hat{\lambda}_{\max }: \hat{A}\right)$, and that vice versa.

Lemma 7 Suppose that $A$ is an irreducible nonnegative matrix whose diagonal entries are all units, and that $\hat{A}$ is defined by (2.5). Let $\lambda_{\max }$ and $\hat{\lambda}_{\max }$ the principal eigenvalue of the matrix $A$ and the principal eigenvalue of the matrix $\hat{A}$, respectively. Then, a positive eigenvector $w$ of $A$ corresponding to $\lambda_{\max }$ is a positive eigenvector $\hat{w}$ of $\hat{A}$ corresponding to $\hat{\lambda}_{\max }$ and vice versa. Furthermore,

$$
M\left(\lambda_{\max }: A\right)=M\left(\hat{\lambda}_{\max }: \hat{A}\right),
$$

and

$$
\hat{\lambda}_{\max }=\frac{\lambda_{\max }-1}{n-1}
$$

Proof: It follows from the definition of $\hat{A}$ that

$$
\hat{A} w=\frac{1}{n \cdot-1}(A w-w)=\frac{\lambda_{\max }-1}{n-1} w .
$$

This means that $w$ and $\left(\lambda_{\max }-1\right) /(n-1)$ are an eigenvector of the matrix $\hat{A}$ and the corresponding eigenvalue, respectively. Since $w$ is positive, it follows from Lemma 5 and Corollary 2 that $w=\mu \hat{w}$ for some positive $\mu$. Dividing (2.10) by the positive number $\mu$, we have $\hat{A} \hat{w}=\left(\lambda_{\max }-1\right) /(n-1) \hat{w}$. Since $\hat{\lambda}_{\max }$ corresponds to the eigenvector $\hat{w}$, it follows 
that $\hat{\lambda}_{\max }=\left(\lambda_{\max }-1\right) /(n-1)$, and that the eigenvector $w$ of the matrix $\hat{A}$ corresponds to the eigenvalue $\hat{\lambda}_{\max }$. Hence $w$ is an eigenvector of $\hat{A}$ corresponding to $\hat{\lambda}_{\max }$. In the same manner, we can show that $\hat{w}$ is the principal eigenvector of the matrix $A$.

Since $\operatorname{dim} M\left(\lambda_{\max }: A\right)=1$, for every nonzero vector $v \in M\left(\lambda_{\max }: A\right)$ there exists a scaler $\mu \neq 0$ such that $v=\mu w$. Since $w$ is an eigenvector of $\hat{A}$ corresponding to $\hat{\lambda}_{\max }$, $v=\mu w$ is also an eigenvector of $\hat{A}$ corresponding to $\hat{\lambda}_{\text {max }}$. In the similar way, we can prove that every nonzero vector $u \in M(\hat{\lambda}: \hat{A})$ is an eigenvector of $A$ corresponding to $\lambda_{\max }$. Hence, $M\left(\hat{\lambda}_{\max }: \hat{A}\right)=M\left(\lambda_{\max }: A\right)$.

Theorem 8 A priority weight vector of EM is an optimal solution of the optimization problem (2.7), and vice versa. A priority weight vector of EM is an optimal solution of the optimization problem (2.8), and vice versa.

Proof: Directly from Lemma 7.

Note that every ratio of a self-evaluation value and the corresponding non-self-evaluation value is equal to the principal eigenvalue of the matrix $\hat{A}$ if and only if a vector of the selfevaluation value is an optimal solution of either the problem (2.7) or (2.8).

We summarize some properties of the principal eigenvector of the matrix $\hat{A}$ as follows: Theorem 9 Suppose that $A$ is a positive matrix with all diagonal elements $a_{i i}=1$, and that $\hat{A}$ is defined by (2.5). Let $\hat{\lambda}_{\max }$ be the principal eigenvalue of the matrix $\hat{A}$. Then, $\hat{\lambda}_{\max }>0$ and there exists a unique positive eigenvector $\hat{w}$ of $\hat{A}$ corresponding to $\hat{\lambda}_{\text {max }}$. Furthermore, for every positive vector $w \notin M\left(\hat{\lambda}_{\max }: \hat{A}\right)$,

$$
\min \left\{\frac{\hat{a}_{1} w}{w_{1}}, \ldots, \frac{\hat{a}_{n} w}{w_{n}}\right\}<\hat{\lambda}_{\max }<\max \left\{\frac{\hat{a}_{1} w}{w_{1}}, \ldots, \frac{\hat{a}_{n} w}{w_{n}}\right\} .
$$

For every positive vector $w \in M\left(\hat{\lambda}_{\max }: \hat{A}\right)$,

$$
\hat{\lambda}_{\max }=\frac{\hat{a}_{1} w}{w_{1}}=\cdots=\frac{\hat{a}_{n} w}{w_{n}} .
$$

Proof: The proof of (2.11) will be given by contradiction. Suppose that there exists a positive $n$-dimensional vector $\bar{w} \notin M\left(\hat{\lambda}_{\max }: \hat{A}\right)$ such that either $\hat{\lambda}_{\max } \leq\left(\hat{a}_{i} \bar{w} / \bar{w}_{i}\right)$ for every $i \in I$ or $\hat{\lambda}_{\max } \geq\left(\hat{a}_{i} \bar{w} / \bar{w}_{i}\right)$ for every $i \in I$. Let $J=\left\{i \in I \mid \hat{\lambda}_{\max }=\left(\hat{a}_{i} \bar{w} / w_{i}\right)\right\}$.

We now consider that $\hat{\lambda}_{\max } \leq\left(\hat{a}_{i} \bar{w} / \bar{w}_{i}\right)$ for every $i \in I$. Since $\bar{w} \notin M\left(\hat{\lambda}_{\max }: \hat{A}\right)$, it follows from Theorem 3 that $J \neq \emptyset$ and $J \neq I$. Therefore there exists an index $l$ such that $\hat{\lambda}_{\max }<\left(\hat{a}_{l} \bar{w} / \bar{w}_{l}\right)$ and we can choose a positive number $\epsilon$ such that $0<\epsilon<\left(\hat{a}_{l} \bar{w} / \hat{\lambda}_{\max }\right)-\bar{w}_{l}$.

Let $\tilde{w}_{i}=\bar{w}_{i}$ for all $i \neq l$ and $\tilde{w}_{l}=\bar{w}_{l}+\epsilon$, then by the choice of $\epsilon>0$ we obtain

$$
\frac{\hat{a}_{l} \tilde{w}}{\tilde{w}_{l}}=\frac{\hat{a}_{l} \bar{w}}{\bar{w}_{l}+\epsilon}>\hat{\lambda}_{\max } \quad \text { and } \quad \frac{\hat{a}_{i} \tilde{w}}{\tilde{w}_{i}}=\frac{\hat{a}_{i} \bar{w}+\hat{a}_{i l} \epsilon}{\bar{w}_{i}}>\frac{\hat{a}_{i} \bar{w}}{\bar{w}_{i}} \geq \hat{\lambda}_{\max } \quad \text { for all } i \neq l
$$

since $\hat{a}_{l i}>0$ for all $i \neq l$ and $\hat{a}_{l l}=0$. This contradicts (2.2) of Theorem 3 .

In the other case where $\hat{\lambda}_{\max } \geq\left(\hat{a}_{i} \bar{w} / \bar{w}_{i}\right)$ for every $i \in I$, we will lead to the contradiction in the same manner and complete the proof of this case.

The validity of (2.12) is immediately followed from Theorem 3.

Theorem 10 Let $A$ and $\hat{\lambda}_{\max }$ be a positive reciprocal matrix and the principal eigenvalue of the matrix $\hat{A}$ defined by (2.5), respectively. Then,

$$
\hat{\lambda}_{\max } \geq 1 \text {. }
$$


Proof: Since the principal eigenvalue of the matrix $A$ is not less than $n$ (see for details [7]), it follows from (2.9) of Lemma 7 that $\hat{\lambda}_{\max } \geq 1$.

\section{An Equilibrium Model Based on Game Theory}

In this section we will discuss our model-based AHP in terms of noncooperative game played by $n$ competitive companies. All $n$ companies develop the new products with the same functions individually and they will sell each product for the common market. The $i^{\text {th }}$ company plans to sell its product by the price $w_{i}$. The consumers of the market have a common pairwise comparison matrix $A=\left[a_{i j}\right]$ with respect to the brand preference of the existing products. They consider that paying $w_{j}$ for the $j^{t h}$ company's product is indifferent to paying $a_{i j} w_{j}$ for the $i^{\text {th }}$ company's product. Suppose that the brand preference table $A$ is not affected by the price vector $w=\left(w_{1}, \ldots, w_{n}\right)^{T}$. The consumers will estimate the price of the $i^{t h}$ company's product as $\hat{a}_{i} w$ after knowing the prices of $(n-1)$ others' companies. The ratio $\left(\hat{a}_{i} w / w_{i}\right)$ represents the gap between the price estimated by the consumers and one determined by the $i^{t h}$ company. When the ratio $\left(\hat{a}_{i} w / w_{i}\right)$ is greater than 1 , the consumers consider that the $i^{\text {th }}$ company's product is relatively inexpensive, and they want to buy the product whose ratio is equal to $\max \left\{\left(\hat{a}_{i} w / w_{i}\right) \mid i \in I\right\}$. On the other hand, all companies want to determine the high price of each product as much as possible. Here, we define

$$
L^{i}(w)=\max \left\{\frac{\hat{a}_{i} w}{w_{i}} \mid i \in I\right\}-\frac{a_{i} w}{w_{i}}
$$

as the loss function of the $i^{\text {th }}$ company. Suppose that every company determines the price in order to avoid its loss quantity as much as possible. We denote this game by $\Gamma[A, L]$.

A price vector $\bar{w}$ in the game $\Gamma[A, L]$ is called an equilibrium price vector if it satisfies

$$
L^{i}(\bar{w}) \leq L^{i}\left(\bar{w}_{1}, \ldots, \bar{w}_{i-1}, w_{i}, \bar{w}_{i+1}, \ldots, \bar{w}_{n}\right),
$$

for all $w_{i}>0$ and for all $i \in I$. In other words, $\bar{w}$ is an equilibrium price vector in $\Gamma[A, L]$ if no company can decrease strictly its current loss by unilaterally changing the funds.

We will now translate the game $\Gamma[A, L]$ into our model-based AHP. The brand preference table and the price vector in the game $\Gamma[A, L]$ correspond to the pairwise comparison matrix and the self-evaluation vector in our model-based AHP, respectively. Determining an equilibrium prices corresponds to evaluating the brand preference order of the consumers. The behavior criteria of each alternative is as follows:

- Each alternative wants possibly to receive the larger non-self-evaluation value in comparison with its self-evaluation value.

- Each alternative considers that the distance from the ratio between its self-evaluation value and the corresponding non-self-evaluation value to the maximum ratio among all alternatives' is the degree of the misjudgment.

Then it wants to decrease its misjudgment. Suppose that the $i^{\text {th }}$ alternative can not decrease its misjudgment degree whatever self-evaluation value it gives to itself under the fixed selfevaluation values of $(n-1)$ other alternatives. Then it will not change the self-evaluation value. When any one of all alternative can not decrease strictly the misjudgment degree by changing its self-evaluation value individually, we consider that the self-evaluation vector reaches an equilibrium state in our model-based AHP.

Theorem 11 Suppose that $A$ is a positive matrix with all diagonal entries $a_{i i}=1$. An equilibrium price vector of $\Gamma[A, L]$ is an eigenvector of $A$ corresponding to the principal eigenvalue, and vice versa. 
Proof: Suppose that $\tilde{w}$ is an eigenvector of $A$ corresponding to the principal eigenvalue. Then we see from Lemma 7 that $\tilde{w}$ is also an eigenvector of $\hat{A}$. It follows from Theorem 9 and the definition of $L^{i}(w)$ that for all $i \in I$

$L^{i}(\tilde{w})=\max \left\{\frac{\hat{a}_{j} \tilde{w}}{\tilde{w}_{j}} \mid j \in I\right\}-\frac{\hat{a}_{i} \tilde{w}}{\tilde{w}_{i}}=0 \leq L^{i}\left(\tilde{w}_{1}, \ldots, \tilde{w}_{i-1}, w_{i}, \tilde{w}_{i+1}, \ldots, \tilde{w}_{n}\right) \quad$ for all $w_{i}>0$.

This means that $\tilde{w}$ is an equilibrium price vector of $\Gamma[A, L]$.

Suppose that a positive vector $\bar{w}$ is not an eigenvector of $A$ corresponding to the principal eigenvalue. Then, we see from Lemma 7 that $\bar{w}$ is neither an eigenvector of $\hat{A}$ corresponding to the principal eigenvalue. It follows from Theorem 9 that there is an index $k$ such that

$$
0<L^{k}(\bar{w})=\max \left\{\frac{\hat{a}_{j} \bar{w}}{\bar{w}_{j}} \mid j \in I\right\}-\frac{\hat{a}_{k} \bar{w}}{\bar{w}_{k}} .
$$

Let $\tilde{w}_{k}=\bar{w}_{k} / 2, \tilde{w}_{i}=\bar{w}_{i}$ for all $i \neq k$, and $\tilde{w}=\left(\tilde{w}_{1}, \ldots, \tilde{w}_{n}\right)^{T}$. Since $\hat{a}_{k k}=0$ and $\tilde{w}_{k}=\bar{w}_{k} / 2$, we obtain $\hat{a}_{k} \tilde{w}=\hat{a}_{k} \bar{w}$ and $\hat{a}_{i} \tilde{w}=\hat{a}_{i} \bar{w}-\left(\hat{a}_{i k} \bar{w}_{k} / 2\right)<\hat{a}_{i} \bar{w}$ for all $i \neq k$. Therefore we see that

$$
\begin{aligned}
L^{k}(\bar{w}) & =\max \left\{\frac{\hat{a}_{j} \bar{w}}{\bar{w}_{j}} \mid j \in I\right\}-\frac{\hat{a}_{k} \bar{w}}{\bar{w}_{k}}=\max \left\{\max \left\{\frac{\hat{a}_{j} \bar{w}}{\bar{w}_{j}} \mid \begin{array}{c}
j \in I \text { and } \\
j \neq k
\end{array}\right\}, \frac{\hat{a}_{k} \bar{w}}{\bar{w}_{k}}\right\}-\frac{\hat{a}_{k} \bar{w}}{\bar{w}_{k}} \\
& >\max \left\{\max \left\{\frac{\hat{a}_{j} \tilde{w}}{\tilde{w}_{j}} \mid \begin{array}{c}
j \in I \text { and } \\
j \neq k
\end{array}\right\}, \frac{\hat{a}_{k} \bar{w}}{\bar{w}_{k}}\right\}-\frac{\hat{a}_{k} \bar{w}}{\bar{w}_{k}} \\
& =\max \left\{\max \left\{\frac{\hat{a}_{j} \tilde{w}}{\tilde{w}_{j}} \mid \begin{array}{c}
j \in I \text { and } \\
j \neq k
\end{array}\right\}-\frac{\hat{a}_{k} \bar{w}}{\bar{w}_{k}}, 0\right\} \\
& \geq \max \left\{\max \left\{\frac{\hat{a}_{j} \tilde{w}}{\tilde{w}_{j}} \mid \begin{array}{c}
j \in I \text { and } \\
j \neq k
\end{array}\right\}-\frac{\hat{a}_{k} \tilde{w}}{\tilde{w}_{k}}, 0\right\} \\
& =\max \left\{\max \left\{\frac{\hat{a}_{j} \tilde{w}}{\tilde{w}_{j}} \mid \begin{array}{c}
j \in I \text { and } \\
j \neq k
\end{array}\right\}-\frac{\hat{a}_{k} \tilde{w}}{\tilde{w}_{k}}, \frac{\hat{a}_{k} \tilde{w}}{\tilde{w}_{k}}-\frac{\hat{a}_{k} \tilde{w}}{\tilde{w}_{k}}\right\} \\
& =\max \left\{\max \left\{\frac{\hat{a}_{j} \tilde{w}}{\tilde{w}_{j}} \mid \begin{array}{c}
j \in I \text { and } \\
j \neq k
\end{array}\right\}, \frac{\hat{a}_{k} \tilde{w}}{\tilde{w}_{k}}\right\}-\frac{\hat{a}_{k} \tilde{w}}{\tilde{w}_{k}}=\max \left\{\frac{\hat{a}_{j} \tilde{w}}{\tilde{w}_{j}} \mid j \in I\right\}-\frac{\hat{a}_{k} \tilde{w}}{\tilde{w}_{k}} \\
& =L^{k}(\tilde{w}) .
\end{aligned}
$$

Hence $\bar{w}$ is not an equilibrium price vector of $\Gamma[A, L]$.

Theorem 11 implies that an equilibrium price vector $\bar{w}$ is a solution of (2.12) and vice versa. Therefore we call (2.12) the equilibrium equation of $\Gamma[A, L]$. Let $\bar{w}_{i}$ and $\hat{a}_{i} \bar{w}$ be the $i^{\text {th }}$ country's input and output, respectively. Every $\Gamma[A, L]$ with $\hat{\lambda}_{\max } \neq 1$ has an excess or a shortage of the total output $\sum_{i=1}^{n} \hat{a}_{i} \bar{w}$ over the total input $\sum_{i=1}^{n} \bar{w}_{i}$ for any equilibrium price vector $\bar{w}$. On the other hand, for a game $\Gamma[A, L]$ with $\hat{\lambda}_{\max }=1$, the total output can be balanced with the total input and each output can be equal to the corresponding input among all companies. We define $\hat{\lambda}_{\max }=1$ in the equilibrium equation of $\Gamma[A, L]$ as the ideal equilibrium value.

\section{Discrepancy-Minimization Models}

Comparing the self-evaluation value with the corresponding non-self-evaluation value, we know whether the alternative evaluates itself well or it is underestimated/overestimated. Hence, we consider that the alternative evaluates itself well if the self-evaluation value is close 
to the corresponding non-self-evaluation value, and that it is overestimated/underestimated if the self-evaluation value is much less/greater than the corresponding non-self-evaluation value.

In this section we will find a self-evaluation vector $w$ such that all alternatives possibly evaluate themselves well. We suppose that a desirable self-evaluation vector $\bar{w}$ has the following property. For all $i \in I$, a self-evaluation value $\bar{w}_{i}$ of the $i^{t h}$ alternative is very close to the corresponding non-self-evaluation value $\hat{a}_{i} \bar{w}$.

In order to measure the degree of difference between a self-evaluation value and the corresponding non-self-evaluation value, we consider two discrepancy indices as follows:

$$
\begin{aligned}
\gamma_{i}(w) & =\frac{\hat{a}_{i} w}{w_{i}}-1 \\
\delta_{i}(w) & =\frac{w_{i}}{\hat{a}_{i} w}-1
\end{aligned}
$$

Both $\gamma_{i}(w)$ and $\delta_{i}(w)$ vanish when the self-evaluation value $w_{i}$ is equal to the corresponding non-self-evaluation value $\hat{a}_{i} w$. In both indices (4.1) and (4.2), the unit '1' is subtracted from the ratios as an ideal value. Since $\left(\hat{a}_{i} w / w_{i}\right)-\gamma_{i}(w)=1$ and $\left(\hat{a}_{i} w / w_{i}\right)\left(1+\delta_{i}(w)\right)=1, \gamma_{i}(w)$ is the additive gap between the ideal value 1 and $\left(\hat{a}_{i} w / w_{i}\right)$, and $\delta_{i}(w)$ is the multiplicative gap between the ideal value 1 and $\left(\hat{a}_{i} w / w_{i}\right)$. (For the gap between the ideal value 1 and the reciprocal $\left(w_{i} / \hat{a}_{i} w\right), \gamma_{i}(w)$ is multiplicative and $\delta(w)$ is additive. Hereafter $\gamma_{i}(w)$ and $\delta_{i}(w)$ are called additive discrepancy and multiplicative discrepancy, respectively.)

We first consider $\gamma_{i}(w)$ as the $i^{\text {th }}$ discrepancy index. If the $i^{\text {th }}$ discrepancy $\gamma_{i}(w)$ vanishes, i.e., $w_{i}=\hat{a}_{i} w$, the $i^{t h}$ alternative evaluates itself well. Let $\gamma(w)=\left(\gamma_{1}(w), \ldots, \gamma_{n}(w)\right)$ and $\|\gamma(w)\|_{\infty}=\max \left\{\left|\gamma_{1}(w)\right|, \ldots,\left|\gamma_{n}(w)\right|\right\}$, then we define a discrepancy minimization problem $P[A, \gamma]$ with index $\gamma$ as follows:

$$
\begin{array}{cl}
\min . & \|\gamma(w)\|_{\infty} \\
\text { s.t. } & w>0 .
\end{array}
$$

In order to find a priority vector such that all the alternatives are evaluated well as much as possible, the problem $P[A, \gamma]$ minimizes the largest additive discrepancy.

Theorem 12 Suppose that $A$ is a positive matrix with all diagonal entries $a_{i i}=1$, and that $\hat{A}$ is defined by (2.5). A positive eigenvector of $A$ corresponding to the principal eigenvalue is an optimal solution of $P[A, \gamma]$, and vice versa.

Proof: Let $\hat{\lambda}_{\max }$ be the principal eigenvalue of $\hat{A}$. Suppose that $\hat{w}$ is a positive eigenvector of $A$ corresponding to the principal eigenvalue. It follows from Lemma 5 and Lemma 7 that $\hat{w}$ is an eigenvector of $\hat{A}$ corresponding to $\hat{\lambda}_{\max }$. Since $P[A, \gamma]$ is equivalently rewritten as $\min _{w>0} \max \left\{\left(\hat{a}_{i} w / w_{i}\right)-1,1-\left(\hat{a}_{i} w / w_{i}\right) \mid i \in I\right\}$, each optimal objective function value of the two problems $\min _{w>0} \max \left\{\left(\hat{a}_{i} w / w_{i}\right)-1 \mid i \in I\right\}$ and $\min _{w>0} \max \left\{1-\left(\hat{a}_{i} w / w_{i}\right) \mid i \in I\right\}$ is a lower bound of $P[A, \gamma]$. This means from Theorem 9 and Lemma 7 that

$$
\begin{aligned}
\max \left\{\min _{w>0} \max \right. & \left.\left\{\frac{\hat{a}_{i} w}{w_{i}}-1 \mid i \in I\right\}, \min _{w>0} \max \left\{1-\frac{\hat{a}_{i} w}{w_{i}} \mid i \in I\right\}\right\} \\
& =\max \left\{\min _{w>0} \max \left\{\frac{\hat{a}_{i} w}{w_{i}} \mid i \in I\right\}-1,1-\max _{w>0} \min \left\{\frac{\hat{a}_{i} w}{w_{i}} \mid i \in I\right\}\right\} \\
& =\left|\hat{\lambda}_{\max }-1\right| \\
& \leq \min _{w>0} \max \left\{\frac{\hat{a}_{i} w}{w_{i}}-1,1-\frac{\hat{a}_{i} w}{w_{i}} \mid i \in I\right\}=\min _{w>0}\|\gamma(w)\|_{\infty}
\end{aligned}
$$




$$
\begin{aligned}
& \leq\|\gamma(\hat{w})\|_{\infty}=\max \left\{\frac{\hat{a} \hat{w}}{\hat{w}_{i}}-1,1-\frac{\hat{a} \hat{w}}{\hat{w}_{i}} \mid i \in I\right\}=\max \left\{\hat{\lambda}_{\max }-1,1-\hat{\lambda}_{\max }\right\} \\
& =\left|\hat{\lambda}_{\max }-1\right|
\end{aligned}
$$

Hence we see that $\left|\hat{\lambda}_{\max }-1\right|=\min _{w>0}\|\gamma(w)\|_{\infty}=\|\gamma(\hat{w})\|_{\infty}$, and that $\hat{w}$ is an optimal solution of $P[A, \gamma]$.

Suppose that a positive vector $\bar{w}$ is not an eigenvector of $A$ corresponding to the principal eigenvalue. Then we see from Lemma 7 that $\bar{w}$ does not belong to $M\left(\hat{\lambda}_{\max }: \hat{A}\right)$. It follows from Theorem 9 that

$$
\begin{aligned}
\max \left\{\frac{\hat{a}_{i} \bar{w}}{\bar{w}_{i}}-1,1-\frac{\hat{a}_{i} \bar{w}}{\bar{w}_{i}} \mid i \in I\right\} & =\max \left\{\max \left\{\frac{\hat{a}_{i} \bar{w}}{\bar{w}_{i}} \mid i \in I\right\}-1,1-\min \left\{\frac{\hat{a}_{i} \bar{w}}{\bar{w}_{i}} \mid i \in I\right\}\right\} \\
& >\max \left\{\hat{\lambda}_{\max }-1,1-\hat{\lambda}_{\max }\right\}=\left|\hat{\lambda}_{\max }-1\right| .
\end{aligned}
$$

This means that $\bar{w}$ is not an optimal solution of $P[A, \gamma]$.

In order to measure the inconsistency of the reciprocal pairwise comparison matrix $A$, Saaty [7] has developed the consistency index $\left(\lambda_{\max }-n\right) /(n-1)$, where $\lambda_{\max }$ is the principal eigenvalue of $A$.

Corollary 13 Suppose that $A$ is a reciprocal positive matrix, and that the matrix $\hat{A}$ is defined by (2.5). Let $\hat{\lambda}_{\max }$ be the principal eigenvalue of the matrix $\hat{A}$. Then the optimal objective function value of Problem $P[A, \gamma]$ is $\hat{\lambda}_{\max }-1$, which is equal to the consistency index of the matrix $A$.

Proof: Let $\lambda_{\max }$ be the principal eigenvalue of the matrix $A$. Since $A$ is a positive reciprocal matrix, it follows from Theorem 10 that $0 \leq \hat{\lambda}_{\max }-1=\left|\hat{\lambda}_{\max }-1\right|$.

We see from the proof of Theorem 12 and Lemma 7 that

$$
\min _{w>0}\|\gamma(w)\|_{\infty}=\left|\hat{\lambda}_{\max }-1\right|=\hat{\lambda}_{\max }-1=\frac{\lambda_{\max }-1}{n-1}-1=\frac{\lambda_{\max }-n}{n-1}
$$

which is equal to the consistency index of the matrix $A$.

We then consider the discrepancy index $\delta_{i}(w)$. If the $i^{\text {th }}$ discrepancy $\delta_{i}(w)$ vanishes, i.e., $w_{i}=\hat{a}_{i} w$, the $i^{\text {th }}$ alternative evaluates itself well. Let $\delta(w)=\left(\delta_{1}(w), \ldots, \delta_{n}(w)\right)$ and $\|\delta(w)\|_{\infty}=\max \left\{\left|\delta_{1}(w)\right|, \ldots,\left|\delta_{n}(w)\right|\right\}$, then we define a discrepancy minimization problem $P[A, \delta]$ with index $\delta$ as follows:

$$
\begin{aligned}
\min . & \|\delta(w)\|_{\infty} \\
\text { s.t. } & w>0 .
\end{aligned}
$$

In order to find a priority vector such that all alternatives are evaluated well as much as possible, the problem $P[A, \delta]$ minimizes the largest multiplicative discrepancy.

Lemma 14 Suppose that $A$ is a positive matrix with all diagonal entries $a_{i i}=1$, and that $\hat{A}$ is defined by (2.5). Let $\hat{\lambda}_{\max }$ be the principal eigenvalue of the matrix $\hat{A}$. Then,

$$
\max _{w>0} \min \left\{\frac{w_{i}}{\hat{a}_{i} w} \mid i \in I\right\}=\left(\min _{w>0} \max \left\{\frac{\hat{a}_{i} w}{w_{i}} \mid i \in I\right\}\right)^{-1}=\hat{\lambda}_{\max }^{-1} .
$$


Proof: Suppose that $\bar{w}$ is an eigenvector of $\hat{A}$ corresponding to the principal eigenvalue $\lambda_{\max }$. It follows from Lemma 6 and Theorem 9 that

$$
\hat{\lambda}_{\max }=\frac{\hat{a}_{1} \bar{w}}{\bar{w}_{1}}=\cdots=\frac{\hat{a}_{n} \bar{w}}{\bar{w}_{n}}=\min _{w>0} \max \left\{\frac{\hat{a}_{i} w}{w_{i}} \mid i \in I,\right\} .
$$

Since an optimal solution of $\max _{w>0} \min \left\{\left(w_{i} / \hat{a}_{i} w\right) \mid i \in I\right\}$ is that of $\min _{w>0} \max \left\{\left(\hat{a}_{i} w / w_{i}\right) \mid i \in\right.$ $I\}$, and vice versa, we obtain

$$
\max _{w>0} \min \left\{\frac{w_{i}}{\hat{a}_{i} w} \mid i \in I\right\}=\frac{\bar{w}_{1}}{\hat{a}_{1} \bar{w}}=\cdots \frac{\bar{w}_{n}}{\hat{a}_{n} \bar{w}}=\hat{\lambda}_{\max }^{-1}=\left(\min _{w>0} \max \left\{\frac{\hat{a}_{i} w}{w_{i}} \mid i \in I\right\}\right)^{-1} .
$$

Theorem 15 Suppose that $A$ is a positive matrix with all diagonal entries $a_{i i}=1$, and that $\hat{A}$ is defined by the equation (2.5). An optimal solution of $P[A, \delta]$ is an eigenvector of $A$ corresponding to the principal eigenvalue, and vice versa.

Proof: Let $\hat{\lambda}_{\max }$ be the principal eigenvalue of $\hat{A}$. Suppose that $\bar{w}$ is a positive eigenvector of $A$ corresponding to the principal eigenvalue. Then we see from Lemma 7 that $\bar{w}$ is also an eigenvector of $\hat{A}$ corresponding to $\hat{\lambda}_{\text {max }}$. Since the problem $P[A, \delta]$ is equivalently rewritten as $\min _{w>0} \max \left\{\left(w_{i} / \hat{a}_{i} w\right)-1,1-\left(w_{i} / \hat{a}_{i} w\right) \mid i \in I\right\}$, both optimal objective function values of the two problems, $\min _{w>0} \max \left\{1-\left(w_{i} / \hat{a}_{i} w\right) \mid i \in I\right\}$ and $\min _{w>0} \max \left\{\left(w_{i} / \hat{a}_{i} w\right)-1 \mid i \in I\right\}$, are a lower bound of $P[A, \delta]$. This means from Theorem 9 , Theorem 10 and Lemma 7 that

$$
\begin{aligned}
& \max \left\{\min _{w>0} \max \left\{1-\frac{w_{i}}{\hat{a}_{i} w} \mid i \in I\right\}, \min _{w>0} \max \left\{\frac{w_{i}}{\hat{a}_{i} w}-1 \mid i \in I\right\}\right\} \\
&=\max \left\{1-\max _{w>0} \min \left\{\frac{w_{i}}{\hat{a}_{i} w} \mid i \in I\right\}, \min _{w>0} \max \left\{\frac{w_{i}}{\hat{a}_{i} w} \mid i \in I\right\}-1\right\} \\
&=\left|1-\frac{1}{\hat{\lambda}_{\max }}\right| \\
& \leq \min _{w>0} \max \left\{\frac{w_{i}}{\hat{a}_{i} w}-1,1-\frac{w_{i}}{\hat{a}_{i} w} \mid i \in I\right\}=\min _{w>0}\|\delta(w)\|_{\infty} \\
& \leq\|\delta(\bar{w})\|_{\infty}=\max \left\{\frac{\bar{w}_{i}}{\hat{a}_{i} \bar{w}}-1,1-\frac{\bar{w}_{i}}{\hat{a}_{i} \bar{w}} \mid i \in I\right\}=\max \left\{\frac{1}{\hat{\lambda}_{\max }}-1,1-\frac{1}{\hat{\lambda}_{\max }}\right\} \\
&=\left|1-\frac{1}{\hat{\lambda}_{\max }}\right| .
\end{aligned}
$$

Hence we see that $\left|1-1 / \hat{\lambda}_{\max }\right|=\min _{w>0}\|\delta(w)\|_{\infty}=\|\delta(\bar{w})\|_{\infty}$, and that $\bar{w}$ is an optimal solution of $P[A, \delta]$.

Suppose that a positive vector $\bar{w}$ is not an eigenvector of $A$ corresponding to the principal eigenvalue. Then we see from Lemma 7 that $\bar{w} \notin M\left(\hat{A}: \hat{\lambda}_{\max }\right)$. It follows from Theorem 9 and Lemma 14 that

$$
\begin{aligned}
\max \left\{1-\frac{\bar{w}_{i}}{\hat{a}_{i} \bar{w}}, 1-\frac{\bar{w}_{i}}{\hat{a}_{i} \bar{w}} \mid i \in I\right\} & =\max \left\{\max \left\{\frac{\bar{w}_{i}}{\hat{a}_{i} \bar{w}} \mid i \in I\right\}-1,1-\min \left\{\frac{\bar{w}_{i}}{\hat{a}_{i} \bar{w}} \mid i \in I\right\}\right\} \\
& =\max \left\{\left(\min \left\{\frac{\hat{a}_{i} \bar{w}}{\bar{w}_{i}} \mid i \in I\right\}\right)^{-1}-1,1-\left(\max \left\{\frac{\hat{a}_{i} \bar{w}}{\bar{w}_{i}} \mid i \in I\right\}\right)^{-1}\right\} \\
& >\max \left\{\frac{1}{\hat{\lambda}_{\max }}-1,1-\frac{1}{\hat{\lambda}_{\max }}\right\}=\left|1-\frac{1}{\hat{\lambda}_{\max }}\right| .
\end{aligned}
$$


This means that $\bar{w}$ is not an optimal solution of $P[A, \delta]$.

We briefly discuss a discrepancy minimization problem other than $P[A, \gamma]$ and $P[A, \delta]$. Instead of the maximum norm used in each objective function of $P[A, \gamma]$ and $P[A, \delta]$, we could use either of the two norms $L_{1}$ and $L_{2}$, which are denoted by $\|\cdot\|_{1}$ and $\|\cdot\|_{2}$, respectively, for both the discrepancy index vectors $\gamma(w)$ and $\delta(w)$. The norms $L_{1}$ and $L_{2}$ are denoted by $\|\cdot\|_{1}$ and $\|\cdot\|_{2}$, respectively.

The following pairwise comparison matrix $\tilde{A}$ given in [8] is an example that the similar assertions to those of Theorem 12 and 15 are not valid for any one of the four problems: $\min _{w>0}\|\gamma(w)\|_{1}, \min _{w>0}\|\gamma(w)\|_{2}^{2}, \min _{w>0}\|\delta(w)\|_{1}$ and $\min _{w>0}\|\delta(w)\|_{2}^{2}$.

$$
\tilde{A}=\left[\begin{array}{ccccc}
1 & 1 / 6 & 1 / 2 & 1 / 9 & 5 \\
6 & 1 & 2 & 1 & 5 \\
2 & 1 / 2 & 1 & 1 & 5 \\
9 & 1 & 1 & 1 & 5 \\
1 / 5 & 1 / 5 & 1 / 5 & 1 / 5 & 1
\end{array}\right]
$$

Let $\bar{w}=(0.0893,0.3287,0.1983,0.3413,0.0424)$ and $\tilde{w}=(0.0819,0.3433,0.2089,0.3241,0.0418)$, which are reported by Saaty [8] as the principal eigenvector of the matrix $\tilde{A}$ and the solution by LLSM, respectively. For the two self-evaluation vectors $\bar{w}$ and $\tilde{w}$, we have $\sum_{i=1}^{n}\left|\left(\hat{a}_{i} \bar{w} / \bar{w}_{i}\right)-1\right|=17.59866>17.53173=\sum_{i=1}^{n}\left|\left(\hat{a}_{i} \tilde{w} / \tilde{w}_{i}\right)-1\right|$ and $\sum_{i=1}^{n}\left|\left(\hat{a}_{i} \bar{w} / \bar{w}_{i}\right)-1\right|^{2}=$ $61.94254>61.91708=\sum_{i=1}^{n}\left|\left(\hat{a}_{i} \tilde{w} / \tilde{w}_{i}\right)-1\right|^{2}$. Therefore the principal eigenvector $\bar{w}$ of the matrix $\tilde{A}$ is an optimal solution for neither $\min _{w>0}\|\gamma(w)\|_{1}$ nor $\min _{w>0}\|\gamma(w)\|_{2}^{2}$.

For the two problems $\min _{w>0}\|\delta(w)\|_{1}$ and $\min _{w>0}\|\delta(w)\|_{2}^{2}$, we have $\sum_{i=1}^{n} \mid\left(\bar{w}_{i} / \hat{a}_{i} \bar{w}\right)-$ $1\left|=3.893739>3.885626=\sum_{i=1}^{n}\right|\left(\tilde{w}_{i} / \hat{a}_{i} \tilde{w}\right)-1 \mid$ and $\sum_{i=1}^{n}\left|\left(\bar{w}_{i} / \hat{a}_{i} \bar{w}\right)-1\right|^{2}=3.302241>$ $3.020684=\sum_{i=1}^{n}\left|\left(\tilde{w}_{i} / \hat{a}_{i} \tilde{w}\right)-1\right|^{2}$. Therefore the principal eigenvector $\bar{w}$ of the matrix $A$ is an optimal solution for neither $\min _{w>0}\|\delta(w)\|_{1}$ nor $\min _{w>0}\|\delta(w)\|_{2}^{2}$.

\section{Conclusions and Further Extensions}

This article has achieved two research objectives that can be summarized as follows: First, this study has developed several meaningful models for the problem that corresponds to EM, by introducing a self-evaluation value and the corresponding non-self-evaluation value. These models have presented logical justifications for using EM in AHP. This study has interpreted Theorem 3(Frobenius' Theorem) as two optimization problems with respect to a self-evaluation vector. Moreover, we have proposed the noncooperative game $\Gamma[A, L]$ and shown that the principal eigenvector of the pairwise comparison matrix $A$ is equal to an equilibrium solution of $\Gamma[A, L]$. This study has introduced the three criteria, $L_{\infty}$, $L_{1}$ and $L_{2}$ norms, and the two discrepancy indices between a self-evaluation value and the corresponding non-self-evaluation value. Two of six optimization problems generated by the combination of the two discrepancy indices and the three criteria have an optimal solution which is equal to the principal eigenvector of the matrix $A$.

Second, this study has shown that the commonly used reciprocity assumption is not necessary in EM. The reciprocity in the pairwise comparison matrix $A$ is not used in the proofs of the core theorems: Theorem 8, 11, 12 and 15. To illustrate this generalization, the role of reciprocity in EM is shown in Theorem 10 and Corollary 13. We have demonstrated that the reciprocity is not necessary to find a priority weight vector such that $A w=\lambda w$ and $w>0$.

As extensions of this study, the following research issues can be explored in the near future: We have to make new discrepancy optimization problems with other indices and 
criteria than described in this study and analyze properties of an optimal solution of them. Especially the relationship between an optimal solution and the principal eigenvector of the matrix $A$ is an important issue. We need to incorporate the constraints requested by decision makers in models and formulates them as an optimization problem and a stationary point problem [17]. These problems must be compared each other from various viewpoints such as a computational complexity of a problem and properties of a priority weight vector.

\section{Acknowledgment}

The authors wish to thank Prof. Yoshitsugu Yamamoto, Prof. Satoru Fujishige and Prof. Jin Yoshimura for careful reading of an earlier version of this paper. The first author is partly supported by Shizuoka University Fund for Engineering Research.

\section{References}

[1] I. Basak and T.L. Saaty: Group decision making using the analytic hierarchy process. Mathematical Computer Modelling,17 (1993) 101-109.

[2] F. Chatelin: Eigenvalue of Matrix (in Japanese) tr. by M. Iri and Y. Iri (SpringerVerlag,Tokyo,1993) (the French original,Masson,Paris,1988).

[3] K.O. Cogger and P.L. Yu: Eigen weight vectors and least distance approximation for revealed preference in pairwise weight ratio. Journal of Optimization Theory and Applications, 46 (1985) 483-491.

[4] G. Crawford and C.A. Williams: A note on the analysis of subjective judgment matrices. Journal of Mathematical Psychology, 29 (1985) 387-405.

[5] S. Furuya: Matrix and Determinant (in Japanese) (Baifu-kan,Tokyo,1957).

[6] P.T. Harker and L.G. Vargas: The theory of ratio scale estimation: Saaty's analytic hierarchy process. Management Science, 33 (1987) 1383-1403.

[7] T.L. Saaty:Analytic Hierarchy Process (McGraw-Hill,New York,1980).

[8] T.L. Saaty: Eigenvector and logarithmic least squares. European Journal of Operational Research, 48 (1990) 156-160.

[9] T.L. Saaty and L.G. Vargas: Comparison of eigenvalue, logarithmic least square and least square methods in estimating ratio. Journal of Mathematical Modelling, $\mathbf{5}$ (1984) 309-324.

[10] T.L. Saaty and L.G. Vargas: Inconsistency and rank preservation. Journal of Mathematical Psychology, 28 (1984) 205-214.

[11] I. Takahashi: Problems in AHP and ANP, II.(in Japanese) Communications of the Operations Research Society of Japan,43 (1998) 100-104.

[12] A. Takayama: Mathematical Economics (Cambridge University Press, Cambridge,1985).

[13] K. Tone and R. Manabe: AHP Case Studies (in Japanese)(Nikkagiken,Tokyo,1990).

[14] K. Tone: A note on group vs. individual decision making in AHP. Abstracts of the Operations Research Society of Japan, Fall Meeting (1994) 36-37.

[15] Y. Wind and T.L. Saaty: Marketing applications of the analytic hierarchy process. Management Science, 26,7 (1980) 641-656.

[16] N. Yamaki and K. Sekitani: Large scale AHP with incomplete information.(in Japanese) RIMS Kokyuroku 1043,(Kyoto,1998) 143-154.

[17] Y. Yamamoto: Fixed point algorithms for stationary point problems. In M. Iri and K. Tanabe(eds.): Mathematical Programming, Recent Developments and Applications (KTK Scientific Publishers, Tokyo,1989) 283-307. 
[18] F. Zahedi: The analytic hierarchy process - a survey of the method and its applications. Interfaces,16 (1986) 96-108.

Kazuyuki Sekitani

Department of Systems Engineering

Shizuoka University

Hamamatsu, Shizuoka, 432-8561

E-mail: sekitani@sys.eng.shizuoka.ac.jp 